\title{
Identifying functional brain abnormalities in migraine and depression comorbidity
}

\author{
Ying Yang ${ }^{1}$, Kai Wei ${ }^{2}$, Hongchun Zhang ${ }^{1}$, Hongyun $\mathrm{Hu}^{1}$, Li Yan ${ }^{1}$, Wei Gui ${ }^{3}$, Ying Liu ${ }^{1}$, Xin Chen $^{2}$ \\ ${ }^{1}$ Department of Radiology, The First Affiliated Hospital of USTC, Division of Life Sciences and Medicine, University of Science and Technology of \\ China, Hefei, China; ${ }^{2}$ Department of Neurology, Fourth Affiliated Hospital of Anhui Medical University, Hefei, China; ${ }^{3}$ Department of Neurology, \\ The First Affiliated Hospital of USTC, Division of Life Sciences and Medicine, University of Science and Technology of China, Hefei, China
}

Contributions: (I) Conception and design: X Chen, Y Liu, Y Yang; (II) Administrative support: X Chen, Y Liu; (III) Provision of study materials or patients: X Chen, W Gui; (IV) Collection and assembly of data: Y Yang, K Wei, H Zhang, H Hu, L Yan; (V) Data analysis and interpretation: Y Yang; (VI) Manuscript writing: All authors; (VII) Final approval of manuscript: All authors.

Correspondence to: Xin Chen. Department of Neurology, Fourth Affiliated Hospital of Anhui Medical University, 100 Huaihai Avenue, Hefei 230000, China. Email: xinchen_n@163.com; Ying Liu. Department of Radiology, The First Affiliated Hospital of USTC, Division of Life Sciences and Medicine, University of Science and Technology of China, Hefei 230001, China. Email: felice828@163.com.

Background: Migraine and major depressive disorder (MDD) are both highly prevalent brain disorders and are often comorbid. However, the common and distinctive neural mechanisms underlying these disorders and the brain function alterations associated with their comorbidity are largely unknown. We aimed to explore the functional abnormalities of the brain associated with the co-occurrence of migraine and depression.

Methods: High-resolution T1-weighted and resting-state functional magnetic resonance images (MRI) were acquired from 93 well-matched patients with comorbid migraine and depression, patients with migraine, patients with MDD, and healthy controls. Voxel-wise analysis of variance (ANOVA) and a twosample $t$-test of multiple functional variables were performed among the groups. Furthermore, correlation analysis was conducted to detect the clinical significance of the altered functional regions in the brain.

Results: Migraine patients with and without depression revealed widely shared regional networks of functional changes. Brain function changes in the right paracentral lobule and fusiform were specific to patients with comorbid migraine and depression $[\mathrm{P}<0.05$, cluster-level familywise error (FWE)-corrected], while changes in the left thalamus, medial orbital of superior frontal gyrus and triangular part of the inferior frontal gyrus were specific to patients with migraine $(\mathrm{P}<0.05$, cluster-level FWE-corrected). Importantly, the brain activity of the right paracentral lobule, left calcarine, and left dorsolateral superior frontal gyrus was associated with emotional symptoms in the pooled migraine data $(\mathrm{P}<0.05)$.

Conclusions: These findings help to identify the neural correlates underlying patients with migraine and those with comorbid migraine and depression. These shared and distinct brain changes could be used as potential image markers to decipher the comorbidity of the 2 disorders.

Keywords: Migraine; major depressive disorder (MDD); comorbidity; functional magnetic resonance imaging (fMRI)

Submitted Jun 25, 2021. Accepted for publication Dec 12, 2021; Published online: 07 Jan 2022.

doi: 10.21037/qims-21-667

View this article at: https://dx.doi.org/10.21037/qims-21-667 


\section{Introduction}

Migraine is a highly prevalent neurological disorder that affects more than $10 \%$ of adults around the world. Women are 3 times more likely to be affected by migraine than men $(1,2)$. It is typically characterized by recurrent attacks of headache, often accompanied by symptoms such as photophobia, phonophobia, nausea, vomiting, aura, and premonitory symptoms, and a postdrome phase after the headache subsides (3). Depression or major depressive disorder (MDD) is a psychiatric disorder characterized by depressed mood, sleep abnormalities, cognitive decline, and vegetative symptoms, with a lifetime prevalence of approximately $20 \%$ (4). The World Health Organization (WHO) has predicted that depression will be the leading cause of disease burden by 2030 (5).

Numerous studies have consistently demonstrated that migraine and depression are often comorbid, leading to more serious symptoms and poor treatment outcomes (6). In addition, longitudinal studies have demonstrated multiple, bidirectional relationships between migraine and depression (6,7). Comorbidity may be predominantly due to common underlying pathophysiologic and genetic mechanisms, including abnormal brain development and brain activity, a shared genetic basis, neurotransmitters and receptors, sex hormones, as well as stress (8). Therefore, due to the multifaceted interplay between migraine and depression, exploring the brain mechanisms associated with these disorders and comorbidity may help to refine clinical diagnosis, treatment, and research.

Resting-state functional magnetic resonance imaging (rs-fMRI) is a non-invasive imaging technique to measure spontaneous brain activity based on the blood-oxygenlevel-dependent (BOLD) signal $(9,10)$. In the last decades, advances in rs-fMRI have enhanced the potential to evaluate aberrations in the static functional activity of different brain regions and networks in patients experiencing migraine and depression, respectively $(11,12)$. A review including 28 articles reported that, compared with healthy controls (HCs), patients in the interictal phase of migraine had alterations of more than 20 functional connectivity (FC) networks (13). A network-based approach revealed that the 3 most relevant networks related to migraine were the pain processing network (PPN), the default mode network $(\mathrm{DMN})$, and the visual network (VN) (14-16). In MDD patients, reproducibility of functional brain alterations with 1,434 individuals reported hypoactivity in the orbitofrontal, sensorimotor, and visual cortices and hyperactivity in the frontoparietal cortices compared to the controls (17). A meta-analysis of large-scale network dysfunction in MDD also revealed abnormal resting-state functional connectivity (rsFC) within and between brain networks involved in internally-oriented (DMN) or externally-oriented [dorsal attention network (DAN)] attention, processing of emotion [affective network (AN), or ventral attention network (VAN)], and goal-directed regulation of these functions [frontoparietal network (FN)] (18). Collectively, alterations in multiple functional areas have been reported in both migraine and MDD. However, few studies have explored the mechanisms of comorbidity between migraine and depression. The use of fMRI to investigate abnormalities in the left medial prefrontal cortex (mPFC) has contributed to determining the common symptoms in migraine and depression (19).

Previous studies have demonstrated that spontaneous neural activity can be reflected by the amplitude of lowfrequency fluctuations (ALFF), fractional amplitude of lowfrequency fluctuations (fALFF), and regional homogeneity (ReHo) in brain disorders (20). In this study, we aimed to identify the potential brain function mechanisms underlying migraine and depression comorbidity using rs-fMRI in 4 groups: patients with migraine, patients with comorbid migraine and depression, patients with MDD, and HCs. First, we explored the group differences in ALFF, fALFF, and ReHo using a voxel-wise one-way analysis of variance (ANOVA) and two-sample $t$-test. Second, we detected the main and interaction effects of migraine and depression by using voxel-wise two-way ANOVA with migraine and depression as the between-subject factors on brain function. Finally, we explored the correlation between fMRI abnormalities and patient performance, including clinical and neuropsychological variables, to evaluate their potential clinical significance. We hypothesize that common and distinct brain function alterations underlie migraine, depression, and comorbid migraine and depression. We present the following article in accordance with the Materials Design Analysis Reporting (MDAR) reporting checklist (available at https://qims.amegroups.com/article/ view/10.21037/qims-21-667/rc).

\section{Methods}

\section{Participants}

A total of 93 patients were randomly enrolled in this study. Patients were recruited from the Neurology Clinic of 
First Affiliated Hospital of Anhui Medical University, the Headache Clinic of First Affiliated Hospital of University of Science and Technology of China (USTC; Anhui Provincial Hospital), and the Hefei Fourth People's Hospital (Anhui Mental Health Center) between March 2019 and October 2020. The study was conducted in accordance with the Declaration of Helsinki (as revised in 2013). The study was approved by the Ethics Committee of First Affiliated Hospital of Anhui Medical University, and informed consent was provided by all participants.

Migraine without aura was diagnosed according to the International Classification of Headache Disorders, $3^{\text {rd }}$ edition (beta version) (ICHD-3 beta) (21). Recurrent headache disorder manifests in attacks lasting 4 to $72 \mathrm{~h}$, with headaches typically characterized by a unilateral location, pulsating quality, moderate or severe intensity, aggravation by routine physical activity, and association with nausea and/or photophobia and phonophobia. The diagnosis of MDD was made according to the Diagnostic and Statistical Manual of Mental Disorders, 5th edition (DSM-5) criteria (22). The inclusion criteria were as follows: (I) 18-55 years of age; (II) right-handed, and (III) of Han ethnicity. The exclusion criteria were as follows: (I) a history of secondary or other concomitant primary headache disorders, or significant neurological and physical diseases; (II) the presence of other psychiatric disorders, such as schizophrenia, bipolar disorder, substance-induced mood disorder, anxiety disorders, or substance abuse or dependence; (III) a history of head injury with loss of consciousness, or (IV) pregnancy or any contraindications for MRI. All patients were evaluated by at least 2 fullyqualified neurologists and 2 fully-qualified psychologists.

\section{Behavior assessment and subgroup division}

Participant demographic information (including age, gender, years of education) were recorded. Migraine family history, migraine duration, Headache Impact Test-6 (HIT-6), Visual Analogue Scale (VAS), and Migraine Disability Assessment (MIDAS) were used to assess the impact of migraine (23-25). The 17-item Hamilton Rating Scale for Depression (HAMD) (26), the 14-item Hamilton Rating Scale for Anxiety (HAMA) (27), and the Beck Depression Inventory, $2^{\text {nd }}$ edition (BDI-II) (28) score were applied for assessing the depression and anxiety status of the patients. Cognitive function was assessed using the Mini-Mental State Examination (MMSE).

The participants were divided into 4 subgroups: patients with comorbid migraine and MDD $\left(M+/ D_{+}, n=23\right)$, migraine patients without depression $\left(M_{+} / D_{-}, n=25\right)$, MDD patients without migraine $(M-/ D+, n=22)$, and HCs (M-/D-, n=23).

\section{The $M+/ D+$ group}

Participants the $M+/ D+$ group were diagnosed with migraine without aura and comorbid depression. The 17-HAMD score of this group was greater than 7 . The patients were medication-naïve or had undergone a washout period of at least 5 half-lives of their previously prescribed medication.

\section{The M+/D- group}

Participants in the M+/D- group were diagnosed with migraine without aura, and had no history of any psychiatric disorders, including MDD or depressive mood. The 17HAMD score of this group was less than 7 . The cases were medication-naive or had undergone a washout period of at least 5 half-lives of their previously prescribed medication.

\section{The M-/D+ group}

Participants in the $M-/ D+$ group were diagnosed with MDD. They had no history of migraine or other types of headache. All patients were receiving their regular antidepressant medications. The 17-HAMD score of this group was greater than 7 .

\section{The M-/D- group}

The HCs in the M-/D- group were volunteers recruited through friends, university students, and hospital workers. They did not have any diagnosis of migraine or depression. They had no family history of migraine and psychiatric disorders, and were not using medications. The 17-HAMD score of this group was less than 7 .

\section{Image acquisition}

The MRI scans were obtained using a 3.0-Tesla MRI system (Discovery GE750w; GE Healthcare, Buckinghamshire, UK) with a 24-channel head coil at the Information Science Center of USTC. High-resolution, three-dimensional (3D), T1-weighted structural images were acquired using a brain volume (BRAVO) sequence with the following parameters: repetition time $(\mathrm{TR})=8.16 \mathrm{~ms}$; echo time $(\mathrm{TE})=3.18 \mathrm{~ms}$; flip angle $(\mathrm{FA})=12^{\circ}$; field of view $(\mathrm{FOV})=256 \mathrm{~mm} \times 256 \mathrm{~mm}$; matrix $=256 \times 256$; slice thickness $=1 \mathrm{~mm}$, no gap; and 188 sagittal slices. Resting-state BOLD data were acquired using 
a gradient-echo single-shot echo planar imaging (GRE-SS$\mathrm{EPI}$ ) sequence with the following parameters: $\mathrm{TR}=2,400 \mathrm{~ms}$; $\mathrm{TE}=30 \mathrm{~ms} ; \mathrm{FA}=90^{\circ} ; \mathrm{FOV}=192 \mathrm{~mm} \times 192 \mathrm{~mm}$; matrix $=64 \times 64$; slice thickness $=3 \mathrm{~mm}$, slice gap $=1 \mathrm{~mm}$; and 217 volumes. All participants were instructed to keep their eyes closed, relax, move as little as possible, and think of nothing in particular during the scanning. None of the participants were excluded for visually-inspected imaging artifacts. All participants were scanned during interictal phases to ensure image quality.

\section{fMRI data preprocessing}

The functional and anatomical data were preprocessed and analyzed using the Statistical Parametric Mapping software (SPM12; http://www.fil.ion.ucl.ac.uk/spm) and the Data Processing and Analysis for Brain Imaging (DPABI_ V3.1_180801; http://rfmri.org/dpabi) in MATLAB (R2011a) software (29). The first 10 volumes for each participant were discarded to allow the signal to reach equilibrium and the participants to adapt to the scanning noise. The remaining images were corrected by slicing time, and realigned. All participants' BOLD data were within the defined motion thresholds (i.e., translational or rotational motion parameters not exceeding $2.5 \mathrm{~mm}$ or $2.5^{\circ}$ ). Several nuisance covariates, including the linear drift, the estimated motion parameters based on the Friston-24 model, the spike volumes with frame-wise displacement $(\mathrm{FD})>0.5$, the white matter signal, and the cerebrospinal fluid signal, were regressed out from the data. The data were then detrended, and bandpass-filtered from 0.01 to $0.1 \mathrm{~Hz}$. In the normalization step, individual structural images were firstly co-registered with the mean functional image, then the transformed structural images were segmented and normalized to the Montreal Neurological Institute (MNI) space using the Diffeomorphic Anatomical Registration Through Exponentiated Lie algebra (DARTEL) technique. Finally, each functional volume was spatially normalized to the MNI space using the deformation parameters estimated during the above step, and resampled into a $3-\mathrm{mm}$ cubic voxel. After spatial normalization, all data sets were smoothed with a 6-mm-full-width at half-maximum (FWHM) Gaussian kernel.

\section{ALFF, fALFF, and ReHo calculation}

The ALFF was computed as the mean power spectrum in a specific low-frequency band $(0.01-0.1 \mathrm{~Hz})(30)$, and the
fALFF was the ratio of the power spectrum in the lowfrequency band $(0.01-0.1 \mathrm{~Hz})$ to that in the entire frequency range (31). The ReHo was calculated as the Kendall's coefficient of concordance (or Kendall's W) of the time course of a given voxel with those of its nearest neighbors (26 voxels) (32). The ALFF, fALFF, and ReHo were calculated using the DPABI software. For standardization purposes, the ALFF, fALFF, and ReHo of each voxel were divided by the global mean value within the whole-brain mask. Finally, the resulting ReHo images were spatially smoothed with a 6-mm FWHM Gaussian kernel.

\section{Statistical analysis}

\section{Demographic and clinical data}

One-way ANOVA, two-sample t-tests, and chi-square tests were conducted using the Statistical Package for the Social Sciences (SPSS) 23.0 software package (IBM Corp., Chicago, IL, USA) to compare the continuous and categorical variables among the groups. The significance was set at $\mathrm{P}<0.05$.

\section{fMRI imaging}

Voxel-wise one-way ANOVA and the two-sample t-test were conducted to explore group differences in the ALFF, fALFF, and ReHo, using SPM 12. Voxel-wise two-way ANOVA, with migraine and depression as the betweensubject factors, was performed on brain function to identify the main and interaction effects of migraine and depression using SPM 12. Comparisons above were corrected using a cluster-level familywise error (FWE) method, resulting in a cluster defining threshold of $\mathrm{P}=0.001$ and a corrected cluster significance of $\mathrm{P}<0.05$. Then, the brain regions identified in the above analyses were extracted as regions of interest (ROIs). Post-hoc tests were then performed on the ROIs using Bonferroni correction $(\mathrm{P}<0.05 / 4=0.0125)$.

\section{The relationship between functional imaging features and clinical variables}

To further detect whether the intrinsic brain activity of the above ROIs correlated with the clinical variables (duration of illness, HIT-6, VAS, MIDAS, HAMD, HAMA, and BDI-II), correlation analyses were conducted. First, the test of homogeneity was performed. Then, Pearson coefficients correlation analysis was conducted when the 2 continuous variables were both normally distributed; otherwise, the Spearman coefficients correlation analysis was conducted. For these correlation analyses, a significant threshold was 
Table 1 Demographic and clinical characteristics for all participants $(\mathrm{n}=93)$

\begin{tabular}{|c|c|c|c|c|c|c|}
\hline Characteristic & $M+/ D-(n=25)$ & $M-/ D+(n=22)$ & $M+/ D+(n=23)$ & $M-/ D-(n=23)$ & $\mathrm{F} / \mathrm{t} / \chi^{2}$ & $P$ value \\
\hline Gender (female/male) & $18 / 7$ & $18 / 4$ & $19 / 4$ & $19 / 4$ & 1.211 & $0.750^{\mathrm{a}}$ \\
\hline Family history of migraine & $17 / 25$ & N/A & $16 / 23$ & $\mathrm{~N} / \mathrm{A}$ & N/A & N/A \\
\hline Duration of illness (months) & $104.40 \pm 61.82$ & $54.12 \pm 84.09$ & $101.22 \pm 64.58$ & $\mathrm{~N} / \mathrm{A}$ & 3.622 & 0.032 \\
\hline $\mathrm{FD}(\mathrm{mm})$ & $0.09 \pm 0.05$ & $0.07 \pm 0.03$ & $0.10 \pm 0.05$ & $0.08 \pm 0.02$ & 2.620 & 0.056 \\
\hline VAS & $6.16 \pm 1.40$ & $\mathrm{~N} / \mathrm{A}$ & $6.22 \pm 1.20$ & N/A & -0.151 & $0.880^{\mathrm{b}}$ \\
\hline MIDAS & $16.76 \pm 10.93$ & $\mathrm{~N} / \mathrm{A}$ & $25.78 \pm 22.73$ & $\mathrm{~N} / \mathrm{A}$ & -1.775 & $0.082^{\mathrm{b}}$ \\
\hline HIT-6 & $59.68 \pm 5.14$ & $\mathrm{~N} / \mathrm{A}$ & $60.91 \pm 4.74$ & $\mathrm{~N} / \mathrm{A}$ & -0.861 & $0.394^{b}$ \\
\hline BDI-II & $5.60 \pm 3.37$ & $\mathrm{~N} / \mathrm{A}$ & $22.39 \pm 5.24$ & $\mathrm{~N} / \mathrm{A}$ & -13.322 & $0.000^{\mathrm{b}}$ \\
\hline
\end{tabular}

The data are shown as the mean $\pm \mathrm{SD} .{ }^{a}$, the $\mathrm{P}$ value was obtained by the chi-square test; ${ }^{\mathrm{b}}$, the $\mathrm{P}$ value was obtained by the two-sample $t$-test; other $\mathrm{P}$ values were obtained by one-way ANOVA; *, $\mathrm{P}<0.05$ obtained by post-hoc analysis (Bonferroni corrected) in pairs of M+/Dand $M-/ D+, M+/ D-$ and $M+/ D+, M-/ D+$ and $M+/ D+, M-/ D+$ and $M-/ D-$, and $M+/ D+$ and $M-/ D-. ~ M+/ D+:$ patients with comorbid migraine and depression; M+/D-: patients with migraine; M-/D+: patients with major depressive disorder; M-/D-: healthy controls. FD, frame-wise displacement; VAS, Visual Analogue Scale; MIDAS, Migraine Disability Assessment; HIT-6, Headache Impact Test; HAMD, Hamilton Rating Scale for Depression; HAMA, Hamilton Rating Scale for Anxiety; BDI-II, Beck Depression inventory-II; ANOVA, analysis of variance; N/A, not available; SD, standard deviation.

set at $\mathrm{P}<0.05$.

\section{Results}

\section{Demographic and clinical characteristics}

Demographic and clinical data of all the participants are listed in Table 1. In this study, the 4 groups were matched for age, gender, education level, and FD $(\mathrm{P}>0.05)$. Significant differences in the scores of HAMD and HAMA were observed among the groups $(\mathrm{P}<0.001)$. Higher scores of HAMD and HAMA were recorded in the groups of $M+$ / $\mathrm{D}+$ and $\mathrm{M}-/ \mathrm{D}+$ than in the groups of $\mathrm{M}+/ \mathrm{D}-$ and $\mathrm{M}-/$ $\mathrm{D}-(\mathrm{P}<0.001)$. Higher scores of BDI-II were recorded in the $M+/ D+$ group than in the $M+/ D-$ group $(\mathrm{P}<0.001)$. There were no significant differences for the scores of VAS, MIDAS, and HIT-6 in the M+/D- group compared with the $M+/ D+$ group $(P>0.05)$.

\section{Differences in ALFF values across groups}

A voxel-wise one-way ANOVA revealed that the intergroup differences in ALFF were mainly located in the right precentral lobule, right paracentral lobule, right inferior occipital gyrus (IOG), right lingual gyrus, right calcarine sulcus, and left thalamus $(\mathrm{P}<0.05$; cluster-level FWEcorrected) (Figure 1A; Table 2). The post-hoc analyses revealed that in the $M+/ D+$ patients, regions of increased ALFF were observed in the right precentral lobule, right paracentral lobule, right IOG, right lingual gyrus, and right calcarine sulcus, relative to the $\mathrm{M}-/ \mathrm{D}$ - participants $(\mathrm{P}<0.05$; Bonferroni corrected). In the $\mathrm{M}+\mathrm{D}-$ participants, regions of increased ALFF were observed in the right precentral lobule, right IOG, right lingual gyrus, right calcarine sulcus, and left thalamus, relative to the M-/D- participants $(\mathrm{P}<0.05$; Bonferroni corrected) (Figure $1 A)$.

A voxel-wise two-sample $t$-test revealed that the $\mathrm{M}+$ / $\mathrm{D}+$ patients had higher ALFF in the right fusiform gyrus, compared with the M-/D- participants $(\mathrm{P}<0.05$; clusterlevel FWE-corrected) (Figure 1B; Table 2).

\section{Differences in $f A L F F$ values across groups}

A voxel-wise one-way ANOVA revealed that the intergroup differences in $\mathrm{fALFF}$ were mainly located in the left superior 

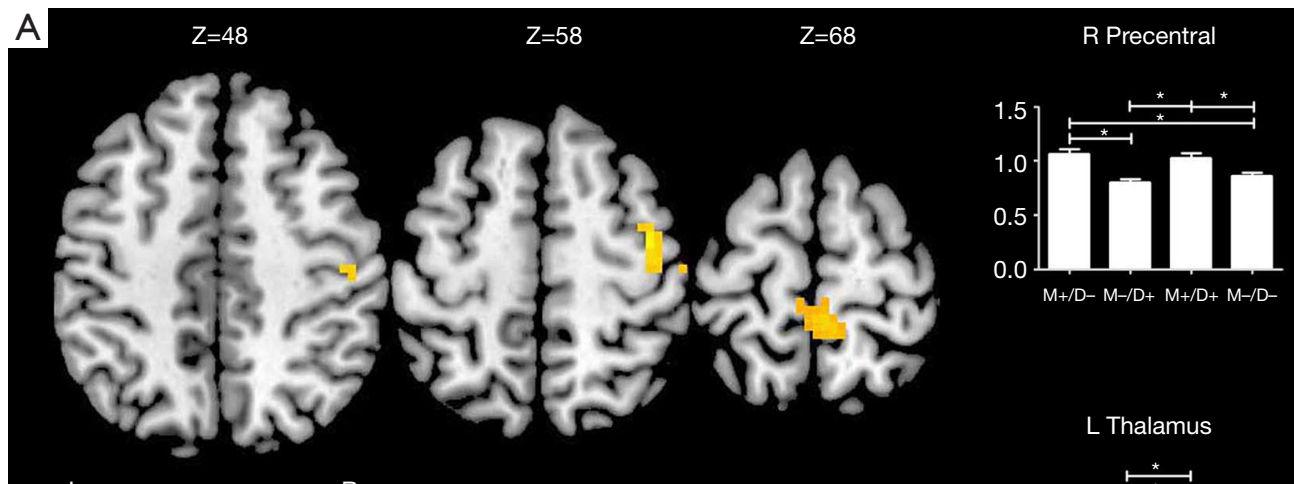

R Paracentral lobule
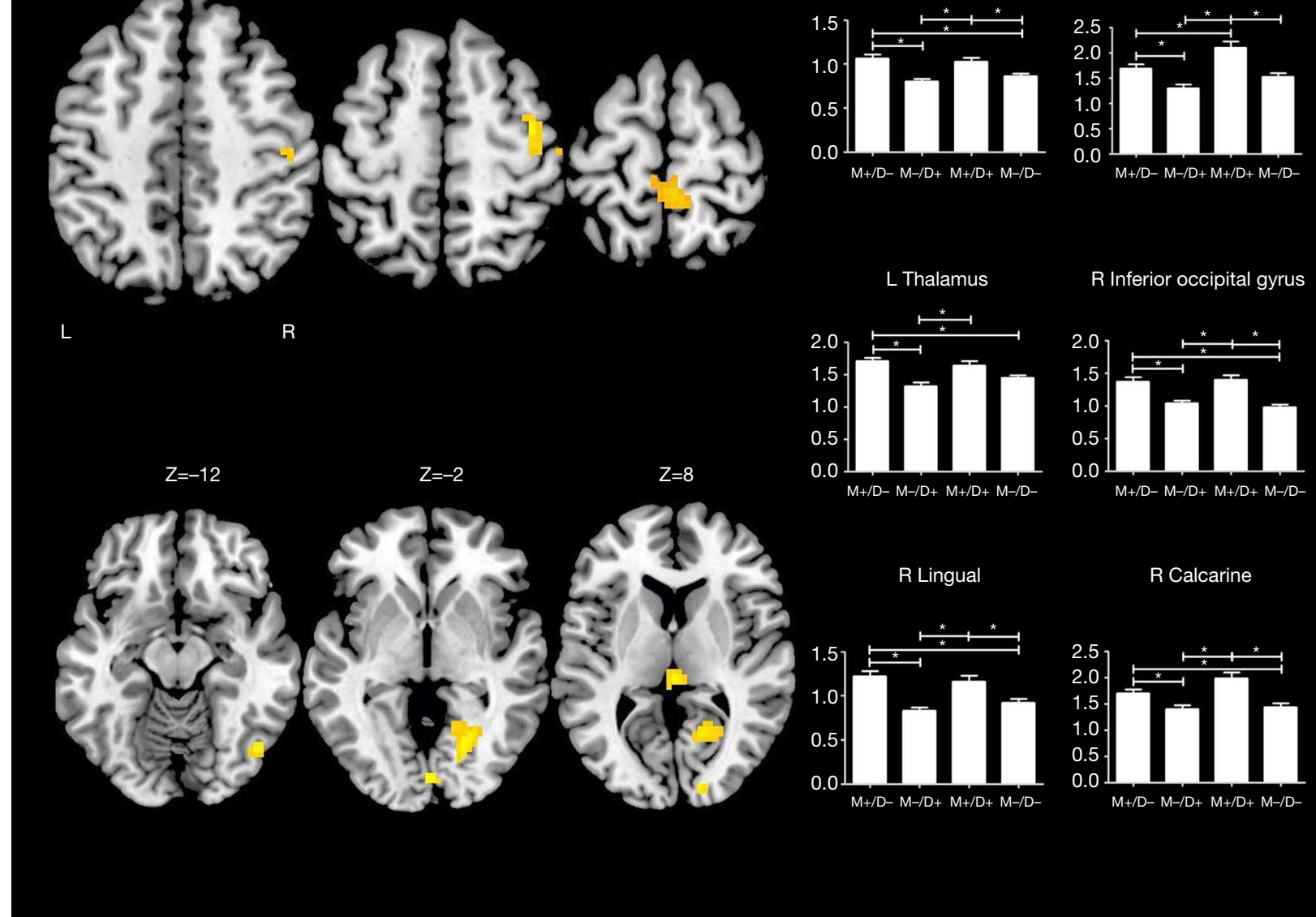

B
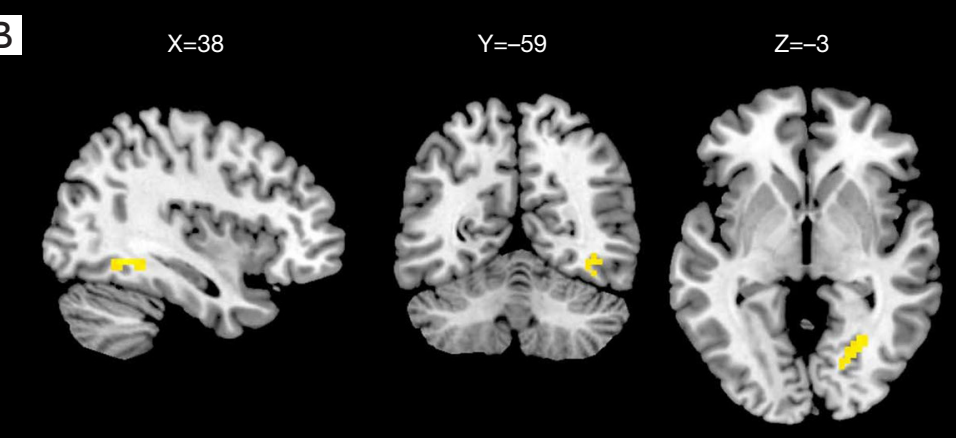

R Fusiform gyrus
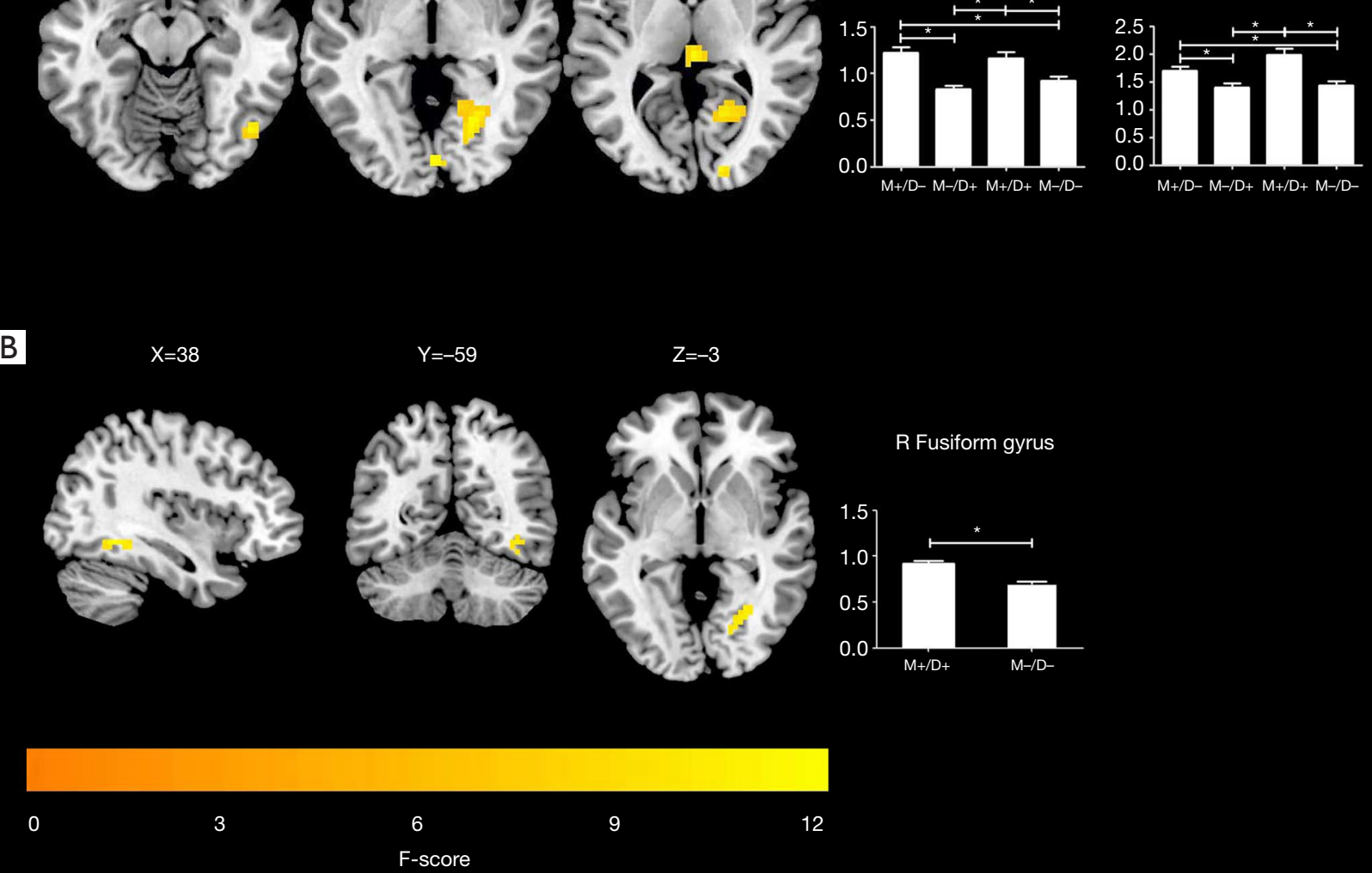

Figure 1 ALFF analysis among the 4 groups. (A) One-way ANOVA analysis on ALFF among the 4 groups. (B) Two-sample t-test on ALFF between M+/D+ and M-/D-. On the right, the histograms exhibit the mean ALFF value by group (error bars represent standard error from the mean) in each identified ROI. *, $\mathrm{P}<0.05$ (Bonferroni-corrected). M+/D+: patients with comorbid migraine and depression; M+/ D-: patients with migraine; M-/D+: patients with major depressive disorder; M-/D-: healthy controls. ALFF, amplitude of low-frequency fluctuations; ROI, region of interest; L, left; R, right; ANOVA, analysis of variance. 
Table 2 Brain areas with significant differences in the ALFF values

\begin{tabular}{lccc}
\hline Clusters & Peak MNI coordinates $(x, y$, and $z)$ & Number of voxels & Max F value \\
\hline ANOVA & $51,-72,-15$ & 31 & 9.7992 \\
R inferior occipital gyrus & $21,-66,-3$ & 141 & 10.7856 \\
R lingual gyrus & $6,-87,0$ & 39 & 8.1106 \\
R calcarine sulcus & $39,-9,57$ & 51 & 10.9265 \\
R precentral lobule & $3,-39,72$ & 68 & 15.5144 \\
R paracentral lobule & $0,-24,12$ & 32 & 9.5223 \\
L thalamus & & & 4.2336 \\
Two-sample $t$-test & $36,-51,-9$ & 46 & \\
R fusiform gyrus & . & & \\
\hline
\end{tabular}

ALFF, amplitude of low-frequency fluctuations; MNI, Montreal Neurological Institute; L, left; R, right; ANOVA, analysis of variance.

frontal gyrus, medial (SFGmed. L) and superior frontal gyri, and dorsolateral superior frontal gyrus (SFGdor. L) ( $\mathrm{P}<0.05$; cluster-level FWE-corrected) (Figure 2A; Table 3). The post-hoc analyses revealed that in the $\mathrm{M}_{+} / \mathrm{D}+$ and $\mathrm{M}_{+} /$ D- participants, regions of decreased fALFF were observed in the SFGmed. L and SFGdor. L, relative to the M-/Dparticipants $(\mathrm{P}<0.05$; Bonferroni corrected) (Figure $2 A)$.

A voxel-wise two-sample $t$-test revealed that the $M_{+} / \mathrm{D}_{-}$ participants had lower fALFF in the left medial orbital of superior frontal gyrus (ORBsupmed. L), and SFGdor. L, compared with the $M-/ \mathrm{D}$ - participants $(\mathrm{P}<0.05$; clusterlevel FWE-corrected) (Figure 2B; Table 3).

\section{Differences in ReHo values across groups}

We discovered that 4 regions, namely, the left IOG, left calcarine sulcus, SFGmed. L, and right lingual gyrus, showed significant ReHo differences among the 4 groups ( $\mathrm{P}<0.05$; cluster-level FWE-corrected) (Figure 3A; Table 4). The post-hoc analyses revealed that in the $M+/ D+$ participants, regions of increased ReHo were observed in the left IOG and left calcarine sulcus, while decreased ReHo was observed in the SFGmed. L, compared to the M-/Dparticipants $(\mathrm{P}<0.05$; Bonferroni corrected). In the $\mathrm{M}+/ \mathrm{D}-$ participants, regions of increased $\mathrm{ReHo}$ were observed in the left IOG, left calcarine sulcus, and right lingual gyrus, while decreased ReHo was observed in the SFGmed. L, compared to the M-/D- participants $(\mathrm{P}<0.05$; Bonferroni corrected) (Figure 3A).

A voxel-wise two-sample t-test revealed that the $\mathrm{M}+\mathrm{D}+$ participants had higher ReHo in the left IOG, compared to the $\mathrm{M}-/ \mathrm{D}-$ participants $(\mathrm{P}<0.05$; cluster-level FWEcorrected) (Figure 3B; Table 4).

\section{Main and interaction effects of migraine and depression on brain function}

As illustrated in Figure 4 and Table 5, a main effect of migraine on the preprocessed function images was observed in the triangular part of the left inferior frontal gyrus (IFGtriang. L) $(\mathrm{P}<0.05$; cluster-level FWE-corrected). Moreover, a significant interaction effect of migraine and depression on the left calcarine sulcus was detected $(\mathrm{P}<0.05$; cluster-level FWE-corrected). Unfortunately, a significant main effect of depression on brain function was not found.

\section{Functional alterations specific to $M+/ D-$}

Combined with the above one-way ANOVA and twosample $t$-test, regions of increased intrinsic activity were observed in the left thalamus, while decreased intrinsic activity was observed in the ORBsupmed. $\mathrm{L}$ in patients with migraine. Furthermore, a main effect of migraine was detected in the IFGtriang. L.

\section{Functional alterations specific to $M+/ D+$}

Combined with the above one-way ANOVA and twosample $t$-test, regions of increased intrinsic activity were observed in the right paracentral lobule and right fusiform gyrus in the participants with comorbid migraine and depression. 


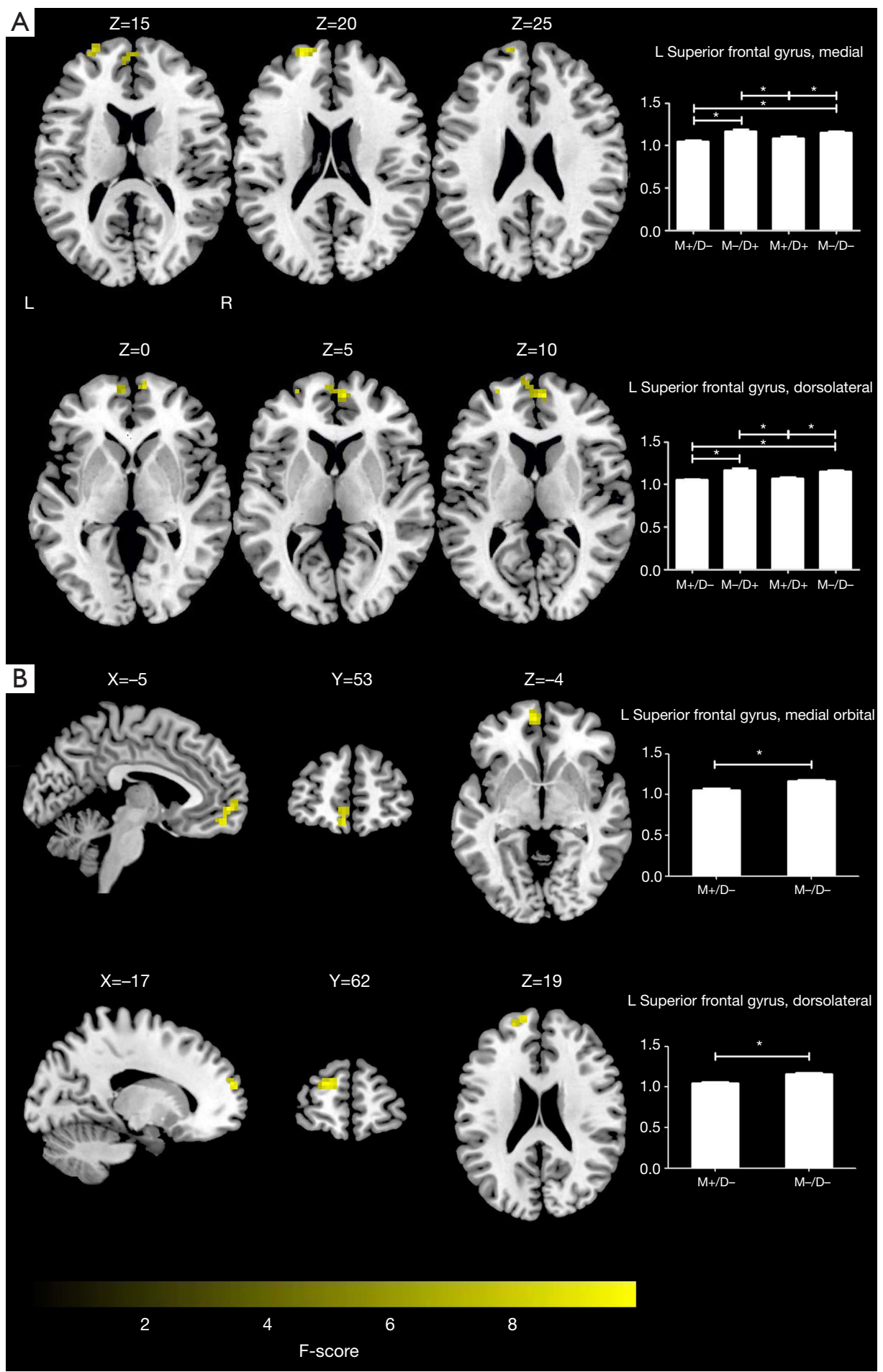

Figure 2 fALFF analysis among the 4 groups. (A) One-way ANOVA analysis on fALFF among the 4 groups. (B) Two-sample $t$-test on fALFF between M+/D- and M-/D-. On the right, the histograms exhibit the mean fALFF value by group (error bars represent standard error from the mean) in each identified ROI. *, $\mathrm{P}<0.05$ (Bonferroni corrected). M+/D+: patients with comorbid migraine and depression; M+/D-: patients with migraine; M-/D+: patients with major depressive disorder; M-/D-: healthy controls. fALFF, fractional amplitude of low-frequency fluctuations; ROI, region of interest; L, left; ANOVA, analysis of variance. 
Table 3 Brain areas with significant differences in the fALFF values

\begin{tabular}{|c|c|c|c|}
\hline Clusters & Peak MNI coordinates (x, y, and z) & Number of voxels & Max $F$ value \\
\hline L superior frontal gyrus, medial & $6,57,9$ & 61 & 9.4713 \\
\hline L superior frontal gyrus, dorsolateral & $-21,60,21$ & 32 & 8.6521 \\
\hline \multicolumn{4}{|l|}{ Two-sample $t$-test } \\
\hline L superior frontal gyrus, dorsolateral & $-15,63,21$ & 29 & 4.6356 \\
\hline
\end{tabular}

fALFF, fractional amplitude of low-frequency fluctuations; MNI, Montreal Neurological Institute; L, left; ANOVA, analysis of variance.

\section{Functional alterations common to $M+/ D-$ and $M+/ D+$ (shared functional alterations)}

Combined with the above one-way ANOVA and twosample $t$-test, regions of increased intrinsic brain activity were observed in the right precentral lobule, right lingual gyrus, bilateral IOG, and calcarine sulcus, while decreased intrinsic brain activity was observed in the SFGmed. L and SFGdor. $\mathrm{L}$ in both the $\mathrm{M}+/ \mathrm{D}-$ and the $\mathrm{M}+\mathrm{D}+$ patients.

\section{Correlation analyses}

In the pooled migraine patients, HAMD (rho $=0.306$; uncorrected $\mathrm{P}=0.035$ ) and $\mathrm{BDI}-\mathrm{II}$ (rho $=0.323$; uncorrected $\mathrm{P}=0.025)$ correlated positively with the ALFF value in the right paracentral lobule (Figure $5 A, 5 B$ ). In addition, the HAMA (rho $=0.29$; uncorrected $\mathrm{P}=0.044$ ) correlated positively with the fALFF value in the SFGdor. L (Figure $5 C$ ). In contrast, the HAMD (rho $=-0.311$; uncorrected $\mathrm{P}=0.032$ ), HAMA (rho $=-0.332$; uncorrected $\mathrm{P}=0.021$ ), and BDI-II scores (rho $=-0.286$; uncorrected $\mathrm{P}=0.048$ ) correlated negatively with the interaction effect of the left calcarine gyrus (Figure $5 D-5 F$ ). None of the other areas with significant changes demonstrated a statistically significant association with the clinical variables.

\section{Discussion}

In the present study, we aimed to explore the brain function abnormalities associated with migraine, depression, and comorbidity of the 2 disorders by using rs-fMRI. First, brain function alterations in the right precentral lobule, right lingual gyrus, bilateral IOG and calcarine sulcus, SFGmed. L, and SFGdor. L were common to patients with migraine and comorbid migraine and depression. Second, patients with comorbid migraine and depression revealed several specific brain areas with functional alterations in the right paracentral lobule and right fusiform gyrus. Third, patients with migraine showed several specific areas with functional alterations in the left thalamus, IFGtriang. L, and ORBsupmed. L. Fourth, the activity in the right paracentral lobule, left calcarine sulcus, and SFGdor. L was correlated with emotional scales in the pooled migraine patients.

Our results showed that patients with comorbid migraine and depression exhibited brain functional alterations in the right precentral, lobule right paracentral lobule, right lingual gyrus, right fusiform gyrus, SFGmed. L, mPFC, SFGdor. L, dorsolateral prefrontal cortex (dlPFC), bilateral IOG, and calcarine sulcus relative to the HCs. Moreover, the interaction of migraine and depression was observed in the left calcarine sulcus. As the anterior node of the DMN, the $\mathrm{mPFC}$ was highly active at rest but inhibited activity mainly engaged in internally-directed cognitions, such as emotional processing and self-referential activity $(33,34)$. A previous study found that migraine and depression jointly affected the development of the left mPFC, which may contribute to determining common symptoms in migraine and depression (19). The dIPFC is a region of the executivecontrol network (ECN) that is most typically associated with executive functions, including working memory and selective attention (35). The precentral gyrus and paracentral lobule, thought to be part of the sensorimotor network $(\mathrm{SMN})$ or PPN, are related to the detection and processing of sensory input (including pain) and the preparation and execution of motor functions $(36,37)$. The lingual gyrus, calcarine sulcus, fusiform gyrus, and IOG are known as brain areas of the $\mathrm{VN}$, that are related to visual perceptual processing and emotion (36). Ma et al. also demonstrated that patients with comorbid migraine and depression had different developmental trajectories in 


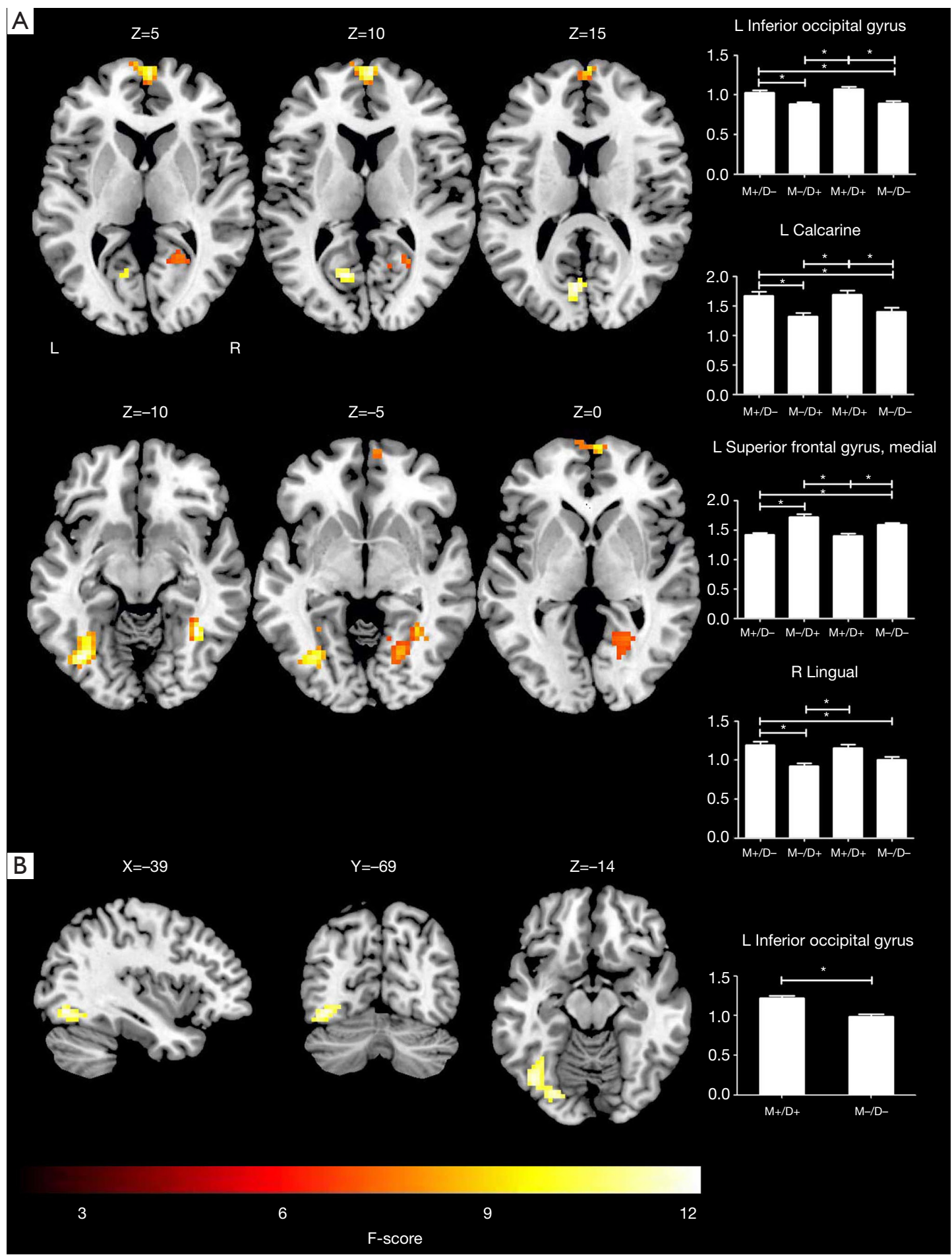

Figure 3 ReHo analysis among the 4 groups. (A) One-way ANOVA analysis on ReHo among the 4 groups. (B) Two-sample $t$-test on fALFF between $M+/ D_{+}$and M-/D-. On the right, the histograms exhibit the mean ReHo value by group (error bars represent standard error from the mean) in each identified ROI. *, $\mathrm{P}<0.05$ (Bonferroni corrected). M+/D+: patients with comorbid migraine and depression; M+/ D-: patients with migraine; M-/D+: patients with major depressive disorder; M-/D-: healthy controls. ReHo, regional homogeneity; ROI, region of interest; L, left; R, right; ANOVA, analysis of variance. 
Table 4 Brain areas with significant differences in the ReHo values

\begin{tabular}{|c|c|c|c|}
\hline Clusters & Peak MNI coordinates ( $x, y$, and $z)$ & Number of voxels & Max F value \\
\hline L inferior occipital gyrus & $-39,-69,-9$ & 72 & 10.3109 \\
\hline L superior frontal gyrus, medial & $3,66,12$ & 85 & 11.4504 \\
\hline L calcarine sulcus & $-6,-75,15$ & 52 & 7.8709 \\
\hline \multicolumn{4}{|l|}{ Two-sample $t$-test } \\
\hline L occipital inferior gyrus & $-30,-72,-6$ & 122 & 4.2754 \\
\hline
\end{tabular}

ReHo, regional homogeneity; MNI, Montreal Neurological Institute; L, left; R, right; ANOVA, analysis of variance.

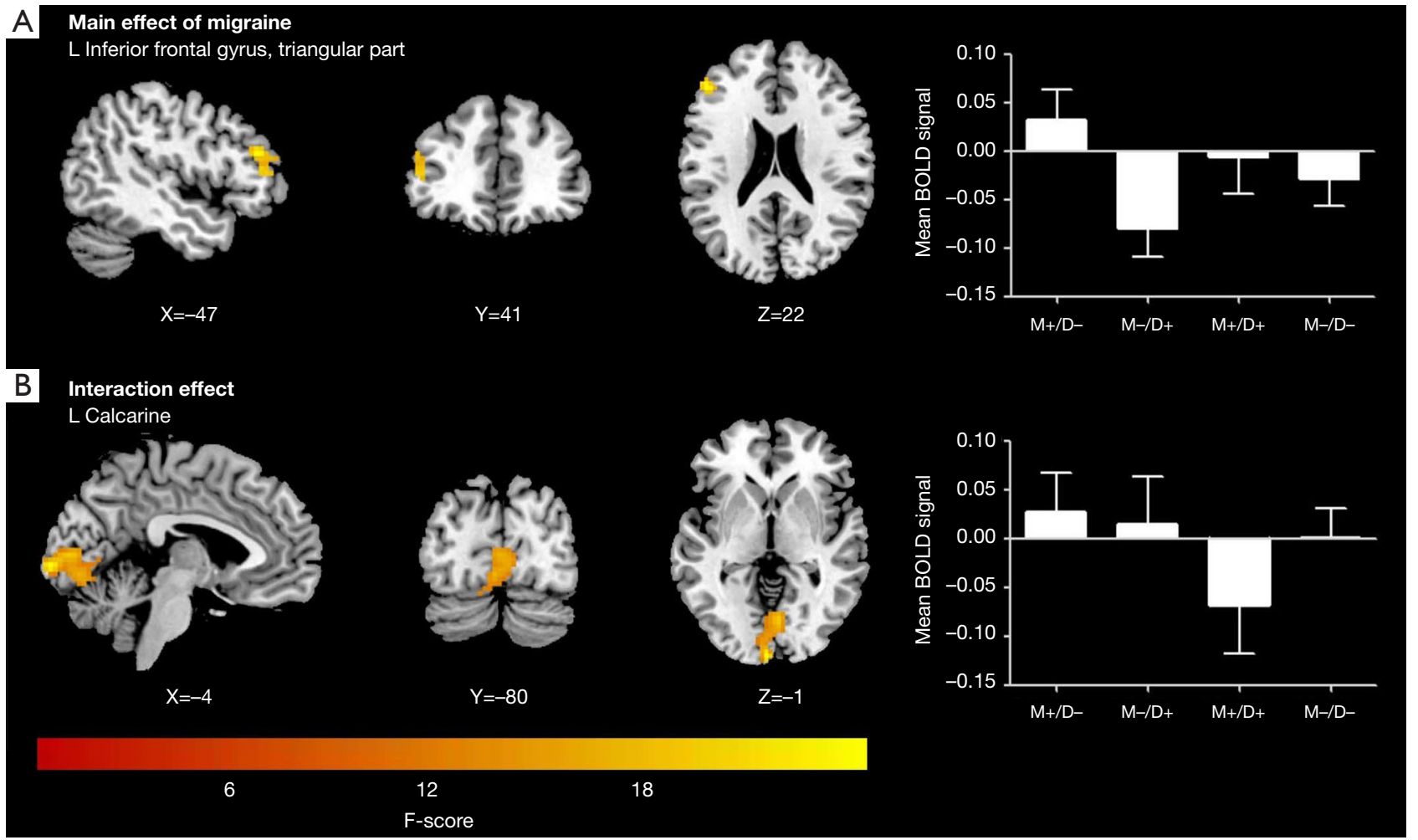

Figure 4 Two-way ANOVA analysis on brain function among the 4 groups. (A) Significant main effect of migraine in the left inferior frontal gyrus, triangular part. (B) Significant interaction effects between migraine and depression in the left calcarine sulcus. On the right, the histogram exhibits the mean BOLD signal by group (error bars represent standard error from the mean). M+/D+: patients with comorbid migraine and depression; M+/D-: patients with migraine; M-/D+: patients with major depressive disorder; M-/D-: healthy controls. L, left; ANOVA, analysis of variance; BOLD, blood-oxygen-level-dependent. 
Table 5 The main and interaction effects of migraine and depression on brain function

\begin{tabular}{|c|c|c|c|}
\hline Clusters & Peak MNI coordinates $(x, y$, and $z)$ & Number of voxels & Max F value \\
\hline L inferior frontal gyrus, triangular part & $-48,36,21$ & 55 & 16.7927 \\
\hline \multicolumn{4}{|c|}{ Interaction effect of migraine and depression } \\
\hline L calcarine sulcus & $-3,-99,0$ & 257 & 22.2694 \\
\hline
\end{tabular}

MNI, Montreal Neurological Institute; L, left.
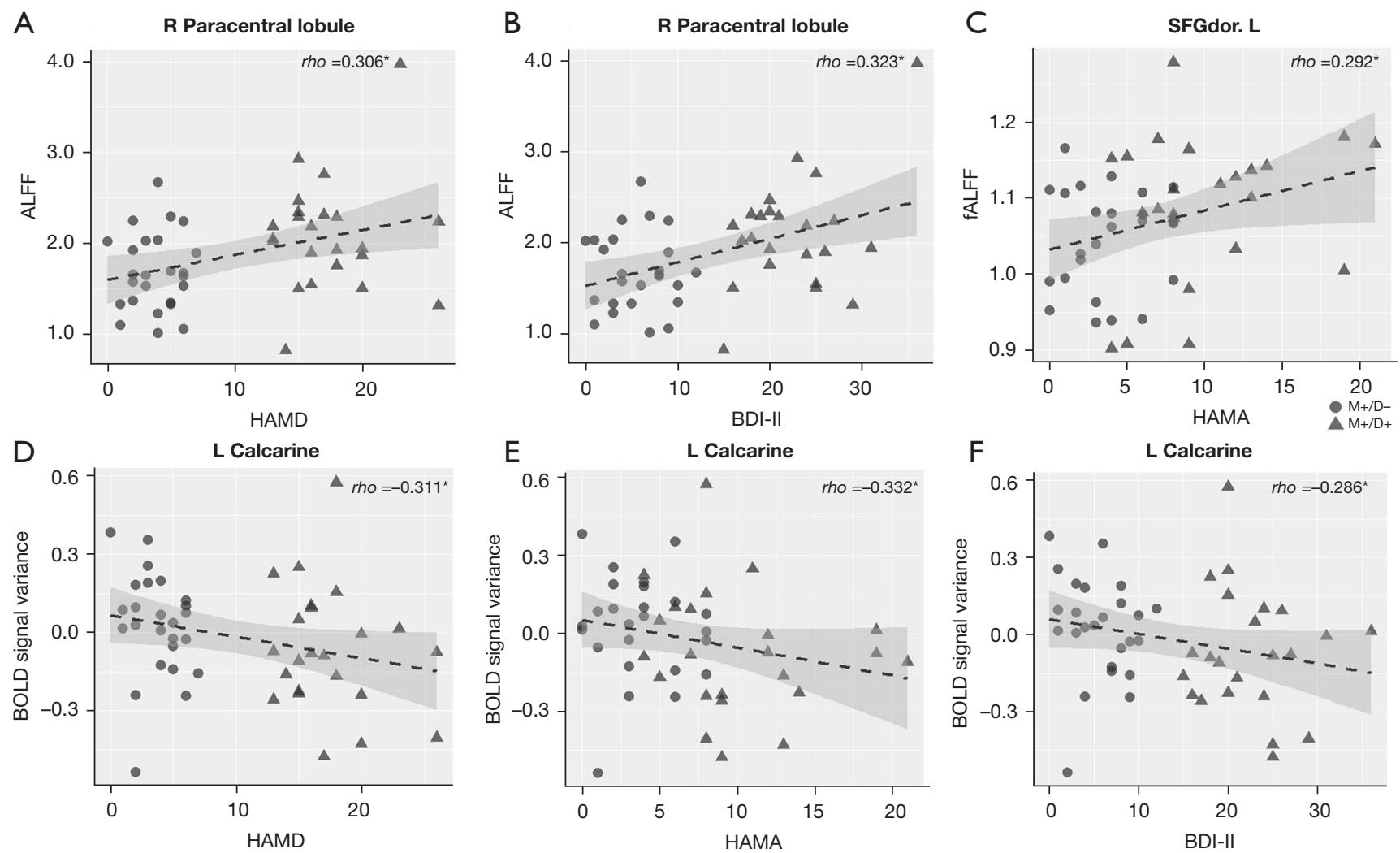

Figure 5 Scatter plots of correlations between brain function and clinical variables in the M+ patients. /D+ and /D- groups are shown separately. (A-C) Positive correlations between brain function alteration (ALFF, fALFF) values and clinical variables (HAMD, BDI-II, and HAMA). (D-F) Negative correlations between BOLD signal variance and HAMD, HAMA, and BDI-II. *, P<0.05. M+/D-: patients with migraine; M+/D+: patients with comorbid migraine and depression. ALFF, amplitude of low-frequency fluctuations; rho, spearman correlation coefficient; HAMD, Hamilton Rating Scale for Depression; HAMA, Hamilton Rating Scale for Anxiety; BDI-II, Beck Depression inventory-II; L, left; R, right; BOLD, blood-oxygen-level-dependent.

the right fusiform gyrus and thalamus, which are associated with transmitting, controlling, and remembering pain and emotion (19). This suggests that the abnormalities observed in in the DMN, ECN, PPN, and VN were profoundly involved in the neuropathological mechanisms of cooccurrence of migraine and depression.
In this study, compared with the HCs, patients with migraine exhibited brain functional alterations in the right precentral lobule, right lingual gyrus, left thalamus, SFGmed. L, SFGdor. L, ORBsupmed. L (mPFC), bilateral IOG, and calcarine sulcus. Furthermore, the main effect of migraine was seen in the IFGtriang. L. It has been suggested 
that the inferior frontal gyrus (IFG), which is part of the VAN, may be related to stimulus-driven attention (38). The thalamus, a region of the PPN, is considered to play an important role in the transmission of nociceptive inputs to cortical pain-related brain regions, including the lateral pathway (in the somatic sensory area) and the medial pathway (in the PFC and limbic system) $(39,40)$. Converging evidence suggests that patients with migraine have multiple brain regions and network alterations, and more than 20 FC networks have been reported, which overlap mostly with our results (13). Thus, the aberrant activity in the DMN, ECN, PPN, VN, and VAN may further suggest the impairment of sensory processing, visual perceptual processing, emotion, and cognitive function (attention, executive, self-related) in migraine patients.

When combing the findings above, we found that regions of functional alterations in the right paracentral lobule and right fusiform gyrus were specific to patients with comorbid migraine and depression, while regions of functional alterations in the left thalamus, IFGtriang. L, and ORBsupmed. $\mathrm{L}$ were specific to patients with migraine. Furthermore, functional alterations in the DMN, ECN, $\mathrm{PPN}$, and $\mathrm{VN}$ were shared by patients with migraine and comorbid migraine and depression.

To further elucidate the association between brain function and clinical variables, observations of the right paracentral lobule, left calcarine sulcus, and SFGdor. $\mathrm{L}$ were correlated with emotional scales in the pooled migraine patients. The paracentral lobule and calcarine sulcus are the initial stage of brain information reception and processing from the periphery, and have been linked to further advanced emotional processing and response $(41,42)$. Previous studies have demonstrated that cognitive control functions mediated by the dIPFC may also relate to emotion, specifically the regulation of negative emotion through reappraisal and suppression strategies (43). The HAMD, BDI-II, and HAMA reflected overall severity of depression and anxiety symptoms in our patients. This result highlighted the roles of the right paracentral, left calcarine sulcus, and SFGdor. L, which were associated with depression and anxiety symptoms in migraine and may even result in comorbidity.

There were several limitations to this study that should be highlighted. First, the sample size was relatively modest, which limits the statistical power for detecting subtle brain alterations, such as the group differences between the $M-/ D+$ group and the HC group and the main effect of MDD. Second, we cannot absolutely rule out the influence of medication and some other detailed clinical data (e.g., migraine frequency) on our results, due to practical and ethical issues. In future studies, we intend to test the reproducibility of our findings by using a larger sample of first-episode, medication-naïve patients. Finally, our crosssectional design did not allow inference on causality. In the future, prospective longitudinal studies should be conducted to examine whether these regional network alterations are a part of the pathogenesis or consequences of the comorbidity.

\section{Conclusions}

In the current study, we identified distinct and shared brain functional alterations in patients with migraine and comorbid migraine and depression. Migraine with and without depression revealed widely shared regional networks of functional changes related to the DMN, PPN, ECN, and VN. In addition, several brain regions were shown to be specific to migraine and comorbidity. Furthermore, our results indicated that the right paracentral lobule, left calcarine sulcus, and SFGdor. L may be the core regions for the development of depression in patients with migraine.

\section{Acknowledgments}

Funding: The work was supported by the National Natural Science Foundation of China (No. 81671290 and No. 82071460) and the 2019 Research Fund Project of Anhui Medical University (No. 2019xkj155).

\section{Footnote}

Reporting Checklist: The authors have completed the MDAR reporting checklist. Available at https://qims.amegroups. com/article/view/10.21037/qims-21-667/rc

Conflicts of Interest: All authors have completed the ICMJE uniform disclosure form (available at https://qims. amegroups.com/article/view/10.21037/qims-21-667/coif). The authors have no conflicts of interest to declare.

Ethical Statement: The authors are accountable for all aspects of the work in ensuring that questions related to the accuracy or integrity of any part of the work are appropriately investigated and resolved. The study was conducted in accordance with the Declaration of Helsinki 
(as revised in 2013). The study was approved by the Ethics Committee of First Affiliated Hospital of Anhui Medical University and informed consent was provided by all participants.

Open Access Statement: This is an Open Access article distributed in accordance with the Creative Commons Attribution-NonCommercial-NoDerivs 4.0 International License (CC BY-NC-ND 4.0), which permits the noncommercial replication and distribution of the article with the strict proviso that no changes or edits are made and the original work is properly cited (including links to both the formal publication through the relevant DOI and the license). See: https://creativecommons.org/licenses/by-nc-nd/4.0/.

\section{References}

1. Ashina M, Katsarava Z, Do TP, Buse DC, Pozo-Rosich P, Özge A, Krymchantowski AV, Lebedeva ER, Ravishankar K, Yu S, Sacco S, Ashina S, Younis S, Steiner TJ, Lipton RB. Migraine: epidemiology and systems of care. Lancet 2021;397:1485-95.

2. Stovner Lj, Hagen K, Jensen R, Katsarava Z, Lipton R, Scher A, Steiner T, Zwart JA. The global burden of headache: a documentation of headache prevalence and disability worldwide. Cephalalgia 2007;27:193-210.

3. Ashina M, Terwindt GM, Al-Karagholi MA, de Boer I, Lee MJ, Hay DL, Schulte LH, Hadjikhani N, Sinclair AJ, Ashina H, Schwedt TJ, Goadsby PJ. Migraine: disease characterisation, biomarkers, and precision medicine. Lancet 2021;397:1496-504.

4. Frankish H, Boyce N, Horton R. Mental health for all: a global goal. Lancet 2018;392:1493-4.

5. WHO. WHO Depression Fact Sheet. Geneva: WHO, 2017.

6. Amoozegar F. Depression comorbidity in migraine. Int Rev Psychiatry 2017;29:504-15.

7. Fugger G, Dold M, Bartova L, Mitschek MMM, Souery D, Mendlewicz J, Serretti A, Zohar J, Montgomery S, Fabbri C, Frey R, Kasper S. Clinical Correlates and Outcome of Major Depressive Disorder and Comorbid Migraine: A Report of the European Group for the Study of Resistant Depression. Int J Neuropsychopharmacol 2020;23:571-7.

8. Zhang Q, Shao A, Jiang Z, Tsai H, Liu W. The exploration of mechanisms of comorbidity between migraine and depression. J Cell Mol Med 2019;23:4505-13.

9. Fox MD, Raichle ME. Spontaneous fluctuations in brain activity observed with functional magnetic resonance imaging. Nat Rev Neurosci 2007;8:700-11.

10. Biswal B, Yetkin FZ, Haughton VM, Hyde JS.

Functional connectivity in the motor cortex of resting human brain using echo-planar MRI. Magn Reson Med 1995;34:537-41.

11. Dichter GS, Gibbs D, Smoski MJ. A systematic review of relations between resting-state functional-MRI and treatment response in major depressive disorder. J Affect Disord 2015;172:8-17.

12. Zou Y, Tang W, Qiao X, Li J. Aberrant modulations of static functional connectivity and dynamic functional network connectivity in chronic migraine. Quant Imaging Med Surg 2021;11:2253-64.

13. Skorobogatykh K, van Hoogstraten WS, Degan D, Prischepa A, Savitskaya A, Ileen BM, Bentivegna E, Skiba I, D'Acunto L, Ferri L, Sacco S, Hansen JM, Amin FM; European Headache Federation School of Advanced Studies (EHF-SAS). Functional connectivity studies in migraine: what have we learned? J Headache Pain 2019;20:108.

14. Coppola G, Di Renzo A, Petolicchio B, Tinelli E, Di Lorenzo C, Serrao M, Calistri V, Tardioli S, Cartocci G, Parisi V, Caramia F, Di Piero V, Pierelli F. Increased neural connectivity between the hypothalamus and cortical resting-state functional networks in chronic migraine. J Neurol 2020;267:185-91.

15. Lee MJ, Park BY, Cho S, Kim ST, Park H, Chung CS. Increased connectivity of pain matrix in chronic migraine: a resting-state functional MRI study. J Headache Pain 2019;20:29.

16. Li Z, Zhou J, Lan L, Cheng S, Sun R, Gong Q, Wintermark M, Zeng F, Liang F. Concurrent brain structural and functional alterations in patients with migraine without aura: an fMRI study. J Headache Pain 2020;21:141.

17. Xia M, Si T, Sun X, Ma Q, Liu B, Wang L, Meng J, Chang M, Huang X, Chen Z, Tang Y, Xu K, Gong Q, Wang F, Qiu J, Xie P, Li L, He Y; DIDA-Major Depressive Disorder Working Group. Reproducibility of functional brain alterations in major depressive disorder: Evidence from a multisite resting-state functional MRI study with 1,434 individuals. Neuroimage 2019;189:700-14.

18. Kaiser RH, Andrews-Hanna JR, Wager TD, Pizzagalli DA. Large-Scale Network Dysfunction in Major Depressive Disorder: A Meta-analysis of Resting-State Functional Connectivity. JAMA Psychiatry 2015;72:603-11.

19. Ma M, Zhang J, Chen N, Guo J, Zhang Y, He L. Exploration of intrinsic brain activity in migraine with 
and without comorbid depression. J Headache Pain 2018;19:48.

20. Yang Y, Zhu DM, Zhang C, Zhang Y, Wang C, Zhang B, Zhao W, Zhu J, Yu Y. Brain Structural and Functional Alterations Specific to Low Sleep Efficiency in Major Depressive Disorder. Front Neurosci 2020;14:50.

21. Headache Classification Committee of the International Headache Society (IHS) The International Classification of Headache Disorders, 3rd edition. Cephalalgia 2018;38:1-211.

22. American Psychiatric Association. Diagnostic and statistical manual of mental disorders, 5th edition (DSM-5). Arlington, VA, USA: American Psychiatric Association, 2013.

23. Yang M, Rendas-Baum R, Varon SF, Kosinski M. Validation of the Headache Impact Test (HIT-6 ${ }^{\text {TM}}$ ) across episodic and chronic migraine. Cephalalgia 2011;31:357-67.

24. Gedikoglu U, Coskun O, Inan LE, Ucler S, Tunc T, Emre U. Validity and reliability of Turkish translation of Migraine Disability Assessment (MIDAS) questionnaire in patients with migraine. Cephalalgia 2005;25:452-6.

25. Gallagher EJ, Liebman M, Bijur PE. Prospective validation of clinically important changes in pain severity measured on a visual analog scale. Ann Emerg Med 2001;38:633-8.

26. Hamilton $M$. Development of a rating scale for primary depressive illness. Br J Soc Clin Psychol 1967;6:278-96.

27. Thompson E. Hamilton Rating Scale for Anxiety (HAM-A). Occup Med (Lond) 2015;65:601.

28. Beck AT, Steer RA, Brown GK. Beck Depression Inventory Second Edition. San Antonio, TX: Psychological Corporation, 1996.

29. Yan CG, Wang XD, Zuo XN, Zang YF. DPABI: Data Processing \& Analysis for (Resting-State) Brain Imaging. Neuroinformatics 2016;14:339-51.

30. Zang YF, He Y, Zhu CZ, Cao QJ, Sui MQ, Liang M, Tian LX, Jiang TZ, Wang YF. Altered baseline brain activity in children with ADHD revealed by resting-state functional MRI. Brain Dev 2007;29:83-91.

31. Zou QH, Zhu CZ, Yang Y, Zuo XN, Long XY, Cao QJ, Wang YF, Zang YF. An improved approach to detection of amplitude of low-frequency fluctuation (ALFF) for resting-state fMRI: fractional ALFF. J Neurosci Methods 2008;172:137-41.

32. Zang Y, Jiang T, Lu Y, He Y, Tian L. Regional homogeneity approach to fMRI data analysis. Neuroimage 2004;22:394-400.

33. Raichle ME. The brain's default mode network. Annu Rev Neurosci 2015;38:433-47.
34. Buckner RL, Andrews-Hanna JR, Schacter DL. The brain's default network: anatomy, function, and relevance to disease. Ann N Y Acad Sci 2008;1124:1-38.

35. Curtis CE, D'Esposito M. Persistent activity in the prefrontal cortex during working memory. Trends Cogn Sci 2003;7:415-23.

36. Barkhof F, Haller S, Rombouts SA. Resting-state functional MR imaging: a new window to the brain. Radiology 2014;272:29-49.

37. Duan G, Chen Y, Pang Y, Feng Z, Liao H, Liu H, Zou Z, Li M, Tao J, He X, Li S, Liu P, Deng D. Altered fractional amplitude of low-frequency fluctuation in women with premenstrual syndrome via acupuncture at Sanyinjiao (SP6). Ann Gen Psychiatry 2021;20:29.

38. Chambers CD, Garavan H, Bellgrove MA. Insights into the neural basis of response inhibition from cognitive and clinical neuroscience. Neurosci Biobehav Rev 2009;33:631-46.

39. Wada A, Shizukuishi T, Kikuta J, Yamada H, Watanabe Y, Imamura Y, Shinozaki T, Dezawa K, Haradome H, Abe O. Altered structural connectivity of pain-related brain network in burning mouth syndrome-investigation by graph analysis of probabilistic tractography.

Neuroradiology 2017;59:525-32.

40. Kupers R, Kehlet H. Brain imaging of clinical pain states: a critical review and strategies for future studies. Lancet Neurol 2006;5:1033-44.

41. Chen C, Jiang WH, Wang W, Ma XC, Li Y, Wu J, Hashimoto K, Gao CG. Impaired visual, working, and verbal memory in first-episode, drug-naive patients with major depressive disorder in a Chinese population. PLoS One 2018; 13:e0196023.

42. Jiang X, Fu S, Yin Z, Kang J, Wang X, Zhou Y, Wei S, Wu F, Kong L, Wang F, Tang Y. Common and distinct neural activities in frontoparietal network in first-episode bipolar disorder and major depressive disorder: Preliminary findings from a follow-up resting state fMRI study. J Affect Disord 2020;260:653-9.

43. Koenigs M, Grafman J. The functional neuroanatomy of depression: distinct roles for ventromedial and dorsolateral prefrontal cortex. Behav Brain Res 2009;201:239-43.

Cite this article as: Yang $\mathrm{Y}$, Wei $\mathrm{K}$, Zhang $\mathrm{H}$, Hu H, Yan L, Gui W, Liu Y, Chen X. Identifying functional brain abnormalities in migraine and depression comorbidity. Quant Imaging Med Surg 2022;12(4):2288-2302. doi: 10.21037/qims-21667 